\title{
Eat, Drink, and Be Merry, for Tomorrow We Diet: Effects of Anticipated Deprivation on Food Intake in Restrained and Unrestrained Eaters
}

\author{
Dax Urbszat, C. Peter Herman, and Janet Polivy \\ University of Toronto
}

\begin{abstract}
This study examined the effect of anticipated food deprivation on intake in restrained and unrestrained eaters. Participants were randomly assigned to a diet condition, in which they expected to diet for a week, or to a control (no-diet) condition. Immediately after being assigned to a condition, participants completed a taste-rating task in which food consumption was measured. Restrained eaters in the diet condition consumed significantly more food than did restrained eaters in the no-diet condition or unrestrained eaters in either condition. Unrestrained eaters consumed the same amount regardless of condition. These results confirm that merely planning to go on a diet can trigger overeating in restrained eaters, reflecting the dynamic connection between dieting and overeating.
\end{abstract}

There seems to be a general consensus in the literature that dieting contributes to binge eating (Bulik, Sullivan, Carter, \& Joyce, 1997; Herman \& Polivy, 1996; Hsu, 1990; Mussell et al., 1997; Nevonen \& Broberg, 2000; Polivy \& Herman, 1985, 1993; Schlundt \& Johnson, 1990; Stice, 2001; Stice \& Agras, 1998; Wilson, 1993). This consensus, however, does not extend to agreement as to precisely which aspects of dieting-a complex of behaviors, motives, cognitions, and emotions-are important in promoting binge eating and how they operate.

For research purposes, dieting has been operationalized in various ways. The Restraint Scale (Herman \& Polivy, 1980) identifies restrained eaters, who attempt to restrict their intake of food in general, and/or of "fattening" food in particular, but who are vulnerable to various factors that disrupt such restriction. The Restraint Scale, in fact, includes some items that assess or presuppose a susceptibility to engage in disinhibited, bingelike eating. Such disinhibited eating is not part of the definition of dieting but appears to be a common enough experience among dieters so that it may be regarded as part of the "dieting syndrome" (Heatherton, Herman, Polivy, King, \& McGree, 1988). The combination of dietary restriction and disinhibition also characterizes many of those persons displaying eating disorders (bulimic and bulimictype anorexic individuals; Polivy \& Herman, 2002). These individuals score high with respect to dietary restraint (Lowe, 2002), and the same dynamics - periods of restricted intake punctuated by bouts of disinhibited eating - are evident in both normal dieters and those with classic eating disorders (Polivy \& Herman, 1993). The intensity of both the restriction and the bingeing may be exaggerated in those with full-blown eating disorders, but the pattern is similar; it appears that the same sort of triggers (e.g.,

Dax Urbszat, C. Peter Herman, and Janet Polivy, Department of Psychology, University of Toronto, Toronto, Ontario, Canada.

This research was supported by grants from the Natural Sciences and Engineering Research Council of Canada and the Social Sciences and Humanities Research Council of Canada.

Correspondence concerning this article should be addressed to C. Peter Herman, Department of Psychology, University of Toronto, Toronto, Ontario M5S 3G3, Canada. E-mail: herman@psych.utoronto.ca emotional upset, or a belief that the diet has been undermined) operate in all cases.

Among the events that precipitate overeating in restrained eaters is the forced ingestion of a high-calorie preload (e.g., a large milkshake). Restrained eaters often eat more following a highcalorie preload than following a low-calorie preload or none at all, whereas unrestrained eaters eat less after a high-calorie preload (Herman \& Polivy, 1996). Even just thinking that one has eaten something fattening - even if the preload is actually low in calories - can serve to disinhibit eating in restrained eaters (Knight \& Boland, 1989; Polivy, 1976; Spencer \& Fremouw, 1979).

Restrained eaters are likely to overeat following the (perceived) ingestion of any "forbidden foods," not just milkshakes (Herman \& Polivy, 1996; Polivy \& Herman, 1985, 1987, 1991; Schlundt \& Johnson, 1990). One possible explanation for this effect is that restrained eaters respond to dietary violations with thoughts ${ }^{2}$ such as "I've blown my diet for today, so I may as well eat what I want now and start my diet again tomorrow." Thus, when restrained eaters are confronted with situations in which they are compelled to consume a forbidden food-as in the case of a forced experimental preload or a social occasion in which forbidden foods are virtually unavoidable - they may conclude that, having already violated their diet, they may as well abandon their customary dietary restraint for the remainder of the day. The conclusion that there is no point to further maintaining dietary restraint once a violation has occurred can lead to overindulgence in foods that are normally not permitted by the diet (Polivy \& Herman, 1991).

Because most diets are structured on a diurnal basis, the dieter tends to evaluate her or his dietary successes and failures in terms of the current day (Herman \& Polivy, in press). Success requires

\footnotetext{
${ }^{1}$ Forbidden foods are defined as those considered by restrained eaters to be inconsistent with their diet owing to their perceived calorie or fat content (e.g., chips, pizza, rich sauces, cake, ice cream, and other rich desserts).

${ }^{2}$ There is no direct evidence for such thought processes in dieters. We can account for their behavior more easily, however, if we posit such thoughts or calculations; it is "as if" the dieter thinks these thoughts. We do not expect dieters to be able to articulate these thoughts.
} 
getting through the day with no violations of the diet. Failure, with a resultant disinhibition of eating, may occur at any point during the day. One consequence of this diurnal approach to dietary success and failure is that even if one fails, at least tomorrow is another day, with a reset dietary clock. This temporal segmentation of dieting does not make much caloric sense because every additional calorie eaten today "carries over" physiologically to tomorrow. The dieter, however, acts as if once her or his diet is "blown" for the day, it matters little if at all how much additional food is consumed. The diet may be resumed the next day with a virtual blank slate.

\section{Anticipated Intake}

If the belief that one's diet has just been compromised leads to overeating, then it follows that the belief that one's diet will soon be compromised may have the same result. Merely anticipating having to eat a forced preload should induce disinhibition because the restrained eater should recognize that a forced preload later in the day undermines the prospect of dietary success for that day just as surely as does a forced preload earlier in the day.

Planning to consume a forbidden food later in the day does lead to overeating in dieters (Ruderman, Belzer, \& Halperin, 1985; Tomarken \& Kirschenbaum, 1984). Presumably, knowing that one's diet will not survive the day makes continued attempts to restrain one's intake useless; the result is a sort of preemptive diet-breaking.

\section{Anticipated Deprivation}

A diet-breaking preload condemns the restrained eater to failure (and disinhibition), whereas the absence of a preload sustains the prospect of success (and continued restriction). If instead of the mere absence of a preload, circumstances dictate a relatively low intake for the remainder of the day, the restrained eater is all the more likely to maintain her or his diet now, even if offered tempting food. The prospect of significant deprivation on future days, however, may have the paradoxical effect of stimulating overeating in the present.

Starting a diet tomorrow is the classic accompaniment of overeating today; however, the converse of this proposition may hold as well: Commitment to dieting tomorrow may contribute to overindulgence today. Lowe (1982) has proposed that the anticipation of future deprivation-usually starting tomorrow-is one of the factors prompting restrained eaters to overeat after a preload. It makes sense-within the always questionable logic of the dieter - to take advantage of an opportunity to load up on excessive quantities of forbidden foods because these foods may not be allowed - certainly not in any quantity - after the diet resumes the next day. Thus, the dieter may reason that it is appropriate to feast now in anticipation of the impending famine. ${ }^{3}$

Lowe (1982) conducted two studies to test the hypothesis that anticipated deprivation can affect the current intake of restrained eaters. The results of these studies were equivocal and did not clearly support Lowe's hypothesis. In defense of the hypothesis, however, it must be noted that the studies had several design features that may have interfered with obtaining the expected effect. Most notably, the anticipated deprivation was only $4 \mathrm{hr}$ in duration and was scheduled for that same day.
Another study (Ruderman et al., 1985) tested Lowe's (1982) hypothesis while attempting to correct the methodological problems suspected to be responsible for the inconsistent results. The results of Ruderman et al.'s (1985) first study did not support the predictions and in some respects were similar to Lowe's. However, although Ruderman's deprivation period extended for $24 \mathrm{hr}$, well into the next day, participants were informed that the deprivation period would conclude with a taste test. The impending second taste test may well have produced disinhibited eating during the first (Day 1) taste test, even among those anticipating deprivation.

Eldredge, Agras, and Arnow (1994) described a last supper effect, "the well-known tendency of some individuals to increase their food consumption ... prior to beginning a diet" (p. 83). Their own study, however, was confined to binge eaters anticipating treatment and lacked a control group; moreover, the dependent measure was weight change over the course of weeks rather than food intake in the period immediately before the onset of a diet.

\section{The Present Study}

The present study attempted to test more directly the last supper effect. To properly test whether anticipated deprivation-independent of anticipated dietary violation-can lead to overeating in restrained eaters, we attempted to create an experimental situation that did not, in and of itself, foster a disinhibition of dietary restraint. Thus, any anticipated dietary violation, such as a taste test of cookies, was scheduled far enough in the future that it would not lead to disinhibited eating in the present. To enhance ecological validity, the anticipated food deprivation was in the form of a diet as opposed to the artificial fasting that has been used in some previous studies. The current literature suggests that one of the crucial elements involved in binge eating is not simply being deprived of food but rather being deprived of certain forbidden foods (Polivy \& Herman, 1993). When participants are led to believe that they will be starting a restrictive diet, the anticipated deprivation becomes more similar to situations facing dieters (and binge eaters) in their normal experience.

With these modifications in mind, we intended this study to test the notion that anticipated deprivation would lead to overeating in restrained eaters. We expected that restrained eaters who believed that they were going to start a week-long restrictive diet would abandon their dietary restraint and consume more food than would restrained eaters who were not anticipating a diet. Moreover, we predicted that unrestrained eaters in both conditions would eat less than would restrained eaters anticipating a diet but more than restrained eaters not anticipating a diet.

\section{Method}

\section{Overview}

Female university students were randomly assigned to the diet or the no-diet condition. Participants in the diet condition agreed to follow a low-fat, calorie-reduced meal plan for a period of 1 week. All participants

\footnotetext{
${ }^{3}$ This logic applies not only to food. "'This could be the last one,' said Darryl Herron, who took hungry drags from a cigarette before trying to go tobacco-free for the next 48 hours" ("Anti-Tobacco Web-Based Show Debuts," Associated Press, May 17, 2001).
} 
completed a taste-rating task in which food consumption was measured. After the taste test, participants completed the Restraint Scale (Herman \& Polivy, 1980).

\section{Participants}

Forty-six female undergraduates at the University of Toronto were recruited for this study in exchange for course credit. On the basis of previous research, those scoring 15 or higher on the Restraint Scale (Herman \& Polivy, 1980) were classified as restrained eaters and those scoring lower than 15 were classified as unrestrained eaters.

\section{Procedure}

Participants agreed to take part in a study investigating the effects of food deprivation on taste perception. On arrival, participants were asked to complete the consent form and were randomly assigned to the diet or no-diet condition. All participants were told that they would perform a taste-perception task and then return in 1 week to perform additional testing. Participants assigned to the diet condition were told that, immediately after the study, they would start a regulated diet for 7 days. These participants were shown the "Canadian Government and University of Toronto approved" student meal plan (see the Appendix). This fictional low-fat, calorie-reduced diet plan consisted of breakfast shakes and prepackaged Lean Cuisine dinners that participants were told would be made available to them free of charge. Participants were told that they would be responsible for their own lunches but that there were specific guidelines laid out by the meal plan regarding which foods they were forbidden to eat. The high-calorie forbidden foods included chips, cookies, ice cream, pizza, chocolate, popcorn, and other "danger foods."

Participants were not actually required to go on the diet; however, it was important that they genuinely believed they would be required to comply with the meal plan for 7 days before returning to the laboratory. Participants assigned to the no-diet condition were told simply that they would return in 7 days to complete further testing.

After being assigned to a condition, participants were asked to complete the State Self-Esteem Scale (Heatherton \& Polivy, 1991) — an adaptation of standard self-esteem scales that provides an assessment of how one feels about oneself at the moment in the domains of appearance, performance, and social competence- and the Positive and Negative Affect Scale (PANAS) - a measure of one's current state on 10 positive and 10 negative affect items (Watson, Clark, \& Tellegen, 1988) - to "get a baseline of how you are feeling right now."

Next, participants were presented with three plates of cookies labeled A, $\mathrm{B}$, and $\mathrm{C}$. The three varieties of cookies were double chocolate, chocolate chip, and mini-Oreos. Along with the three plates of cookies, participants were given three taste-rating forms and a glass of water. Participants were asked to rate each cookie type on the dimensions listed on the rating forms (e.g., bitter, sweet). They were instructed to have as many cookies as was necessary to achieve accurate ratings, which would provide a baseline measure of taste perception against which to compare ratings to be conducted after the 7-day diet (or no-diet) period. Participants were told:

This is a standardized task so you will be given a full 10 minutes to complete it. If you are done early, please feel free to help yourself to cookies-in fact, we have tons-but just make sure that you don't change any of your taste ratings.

When the taste-rating task was completed, the plates of cookies were removed and measured to determine the amount (in grams) of cookies consumed. Participants were then asked to complete the Restraint Scale (Herman \& Polivy, 1980). As a manipulation check, participants in the diet condition were asked to rate the likelihood that they would stick to the diet and how much weight they thought they would lose during the week.
After completing the study, participants were thoroughly debriefed about the purpose of the study and told that they would not be required to actually go on any meal plan or return to do another taste test. The use of deception in this study was explained, and all questions about the study were answered.

\section{Results}

\section{Participants}

Of the 23 participants assigned to the diet condition, 4 declined to go on the meal plan for one week. ${ }^{4}$ Of these, 2 participants were classified as unrestrained eaters and the other 2 as restrained eaters. These 4 participants were run through the no-diet condition but were eliminated from the data analysis because their self-selection into the no-diet condition compromised random assignment. One other participant was eliminated from the study because she "did not like cookies," so there were no intake data for this participant. The final number of participants in the various cells was as follows: no-diet condition, unrestrained eaters, $n=10$, restrained eaters, $n=9$; diet condition, unrestrained eaters, $n=14$, restrained eaters, $n=8$.

\section{Manipulation Check}

As a manipulation check, participants in the diet condition were asked to indicate the extent to which they believed they would be able to stick to the diet. On a scale ranging from 1 to $5(1=$ not at all, $5=$ completely), the overall mean $(M=3.68, S D=.89)$ suggests that participants believed that they would be able to stick to the week-long diet relatively well. It is interesting that the means for restrained eaters $(M=3.67, S D=1.03)$ and unrestrained eaters $(M=3.69, S D=.85)$ were virtually identical.

\section{Main Analyses}

A 2 (restrained vs. unrestrained) $\times 2$ (diet vs. no diet) analysis of variance (ANOVA) was performed on the total grams of cookies consumed. The main effect for condition was significant, $F(1$, $37)=7.7, p<.01, r=.42 .{ }^{5}$ Participants in the diet condition ate more $(M=31.32, S D=16.12)$ than did participants in the no-diet condition $(M=20.84, S D=13.33)$. However, this effect was carried by the restrained eaters, as is reflected in the significant interaction between restraint and diet condition, $F(1,37)=16.64$, $p<.0001, r=.56$. Restrained eaters in the diet condition ate significantly more $(M=44.25, S D=15.98)$ than did restrained eaters in the no-diet condition $(M=14.33, S D=7.89)$ and more than did unrestrained eaters in both the diet condition $(M=23.93$, $S D=10.98)$ and the no-diet condition $(M=26.7, S D=14.82)$, ts (37) $>2, p<.05, r \mathrm{~s}>.31$. Consumption of cookies by

\footnotetext{
${ }^{4}$ We were surprised at how many participants were willing to go on a diet for one week. This willingness can be taken as a reflection of how accepted dieting is in this population.

${ }^{5}$ We have selected $r$ as our measure of effect size, following the recommendation of Rosenthal and DiMatteo (2001).
} 


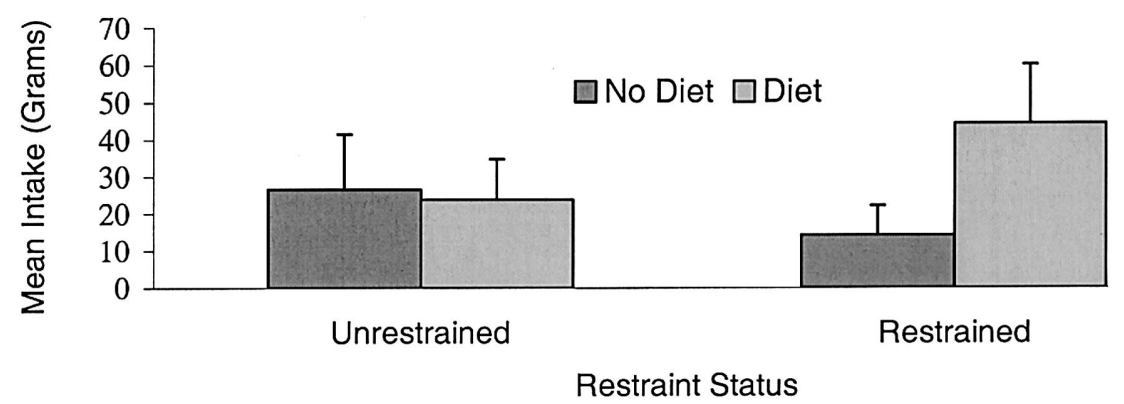

Figure 1. Mean intake (grams) in restrained and unrestrained eaters as a function of diet/no-diet condition. Error bars depict standard errors of the means.

unrestrained eaters in the diet and no-diet conditions did not differ. $^{6}$

Restrained eaters in the no-diet condition ate significantly less than did restrained eaters in the diet condition and unrestrained eaters in the no-diet condition, $t \mathrm{~s}(37)>2, p \mathrm{~s}<.05, r \mathrm{~s}>.31$. However, restrained eaters in the no-diet condition did not eat significantly less than did unrestrained eaters in the diet condition, $t(37)=1.8, r=.28($ see Figure 1$)$.

\section{State Self-Esteem}

Total state self-esteem was analyzed in a 2 (restrained vs. unrestrained) $\times 2$ (diet vs. no diet) ANOVA. The main effect for restraint was significant, $F(1,37)=6.95, p<.05, r=.40$. Restrained eaters across both conditions reported lower total state self-esteem $(M=70.18, S D=12.83)$ than did unrestrained eaters $(M=78.2, S D=8.22)$. Restrained and unrestrained eaters' self-esteem ratings were not significantly affected by experimental condition.

\section{Mood}

A $2 \times 3$ multivariate analysis of variance (MANOVA) was performed on the PANAS mood scale. Main effects and the interaction between restraint and diet condition did not reach significance. Thus, no univariate analyses were conducted.

\section{Discussion}

The data clearly supported the prediction that restrained eaters in the diet condition would consume the most cookies. When the restrained eaters were told that they would begin a week-long, low-calorie diet starting immediately after their participation in a cookie taste-rating task, they ate more cookies than did restrained eaters not asked to diet or unrestrained eaters in either condition. Restrained eaters in the no-diet condition restricted their eating, as expected; they consumed the fewest cookies during the taste test. Cookie consumption by unrestrained eaters did not differ in the diet and no-diet conditions; unrestrained eaters' current eating was unaffected by anticipated deprivation.

The data suggest that anticipation of deprivation (in the form of a low-calorie or restrictive diet) can act as a disinhibitor, causing the restrained eater to overeat when presented with salient forbidden food. In other words, merely expecting to begin a diet in the near future can trigger overeating of foods currently available. It makes some intuitive sense that restrained eaters might take the opportunity to load up on forbidden food (especially when it is salient and palatable) because they will not be allowed or allow themselves to eat such foods once they start their diet. Thus, restrained eaters may reason that it is appropriate to feast now and get their fair share of eating enjoyment before a fairly lengthy and restrictive dietary regime begins. This phenomenon corresponds to Eldredge et al.'s (1994) last supper effect, which they tested in binge eaters. We concur with Eldredge et al. that binge eaters may use the anticipation of a lengthy period of deprivation as a suitable justification for overindulgence.

This disinhibition of eating did not occur in unrestrained eaters. Even when they were asked to go on a diet in the name of science, unrestrained eaters - who would not usually go on a diet- did not show the same tendency to stock up on available food. For unrestrained eaters, intake of forbidden food is not the same hedonically powerful experience that it is for restrained eaters, so perhaps they are less inclined to indulge themselves with a food reward in anticipation of impending suffering. Moreover, unrestrained eaters have probably not had the same extensive experience that restrained eaters have had with the cravings and feelings of deprivation that emerge during restrictive diets. Thus, unrestrained eaters may not anticipate as much suffering in the first place, which would further reduce their motivation to indulge themselves.

The present study confirms that restrained eating may be disinhibited not only by the ingestion of high-calorie, forbidden foods but by the prospect of not being able to ingest high-calorie, forbidden foods over the period of a week. It is no wonder, then, that diets are so fragile; they can be broken not only by eating forbidden food but also by the prospect of not being able to eat forbidden food.

The mood and self-esteem data suggest that the anticipated deprivation effect is mediated more by cognition than by emotion. Future research might well examine more closely exactly what thought processes obtain in the restrained eater about to start a diet. For example, before starting a diet, do restrained eaters reason that they should fill up on salient forbidden foods because they will be

\footnotetext{
${ }^{6}$ Linear regression analysis revealed that restraint score was a significant predictor of food intake in the diet condition, adjusted $R^{2}=3.19, F(1$, $20)=10.83, p<.005, r=.59$, but not in the no-diet condition.
} 
restricted from eating them in the future, or do they think that they can overeat now because they are going to diet in the near future to compensate? This distinction is a subtle one, corresponding to the difference between (a) current indulgence as a compensation for future deprivation and (b) future deprivation as a compensation for current indulgence. Although it would be interesting to explore such subtleties in the thinking patterns that contribute to anticipatory overindulgence, we are by no means certain that restrained eaters are capable of articulating their reasoning with sufficient clarity.

The extent to which anticipated deprivation contributes to the etiology and perpetuation of binge eating is another question that should be examined. There is a general consensus in the literature that dieting contributes to binge eating (see references above); however, by and large, the assumption has been that bingeing is a reaction to the dieting that precedes it. The present study suggests that bingeing may occur in anticipation of the dieting that follows it.

Finally, it is worth pointing out that the anticipation of restricted eating does not inevitably produce disinhibition. After all, restrained eaters whose diets are intact and who are not thinking beyond the end of the current day tend to maintain their restricted intake. This is the basic finding in restrained eaters who have not been given a forced preload and who do not anticipate one (see Herman \& Polivy, 1996, for a review). In effect, they are expecting to diet for the rest of the day, and this expectation does not disinhibit their eating; if anything, it reinforces their restraint. The expectation of dieting for the rest of the day and then again tomorrow (and for the rest of the week), however, seems to have quite a different effect. We are left with the question: What is it about anticipating a diet that promotes disinhibition? Is it that the restrained eater will be dieting tomorrow and that dieting tomorrow is associated with overindulgence today $?^{7}$ Or is it that the restriction on eating was imposed externally, eliciting reactance (Brehm, 1966)? In other words, would disinhibition have occurred if the restriction applied only for the rest of the current day but had been imposed externally? In the present study, we cannot discriminate between these possibilities. Moreover, we must acknowledge the possibility that the dynamics of restraint and disinhibition that we have documented here might depend not only on the specifics of our procedure but also on the particular sample of participants (undergraduate women) in our study. What is clear, in any case, is that fully elucidating the connection between anticipated deprivation and current eating will require additional work.

\footnotetext{
${ }^{7}$ Of course, we did not impose a diet just for tomorrow but rather for the next week. Would an imposed diet extending only through the end of the following day have produced the same effect as we found, or is the extent of disinhibition proportional to the length of the anticipated diet?
}

\section{References}

Brehm, J. W. (1966). A theory of psychological reactance. New York: Academic Press.

Bulik, C. M., Sullivan, P. F., Carter, F. A., \& Joyce, P. R. (1997). Initial manifestations of disordered eating behavior: Dieting versus binging. International Journal of Eating Disorders, 28, 195-201.
Eldredge, K. L., Agras, W. S., \& Arnow, B. (1994). The last supper: Emotional determinants of pretreatment weight fluctuation in obese binge eaters. International Journal of Eating Disorders, 16, 83-88.

Heatherton, T. F. Herman, C. P., Polivy, J., King, G. A., McGree, S. T. (1988). The (mis)measurement of restraint: An analysis of conceptual and psychometric issues. Journal of Abnormal Psychology, 97, 19-28.

Heatherton, T. F., \& Polivy, J. (1991). Development and validation of a scale for measuring self-esteem. Journal of Personality and Social Psychology, 60, 895-910.

Herman, C. P., \& Polivy, J. (1980). Restrained eating. In A. Stunkard (Ed.), Obesity (pp. 208-225). Philadelphia: W. B. Saunders.

Herman, C. P., \& Polivy, J. (1996). What does abnormal eating tell us about normal eating? In H. Meiselman \& H. MacFie (Eds.), Food choice, acceptance, and consumption (pp. 207-238). London: Blackie Academic \& Professional.

Herman, C. P. \& Polivy, J. (in press). Dieting as an exercise in behavioral economics. In G. Loewenstein, D. Read, \& R. F. Baumeister (Eds.), Time and decision. New York: Russell Sage Foundation.

Hsu, L. K. G. (1990). Eating disorders. New York: Guilford Press.

Knight, L. J., \& Boland, F. J. (1989). Restrained eating: An experimental disentanglement of the disinhibiting variables of perceived calories and food type. Journal of Abnormal Psychology, 98, 499-503.

Lowe, M. G. (1982). The role of anticipated deprivation in overeating. Addictive Behaviors, 7, 103-112.

Lowe, M. G. (2002). Dietary restraint and overeating. In C. G. Fairburn \& K. D. Brownell (Eds.), Eating disorders and obesity: A comprehensive handbook (2nd ed., pp. 88-92). New York: Guilford Press.

Mussell, M. P., Mitchell, J. E., Fenna, C. J., Crosby, R. D., Miller, J. P., \& Hoberman, H. M. (1997). A comparison of onset of binge eating versus dieting in the development of bulimia nervosa. International Journal of Eating Disorders, 21, 353-360.

Nevonen, L., \& Broberg, A. G. (2000). The emergence of eating disorders: An exploratory study. European Eating Disorders Review, 8, 279-292.

Polivy, J. (1976). Perception of calories and regulation of intake in restrained and unrestrained subjects. Addictive Behaviors, 1, 237-244.

Polivy, J., \& Herman, C. P. (1985). Dieting and bingeing: A causal analysis. American Psychologist, 40, 193-201.

Polivy, J., \& Herman, C. P. (1987). The diagnosis and treatment of normal eating. Journal of Consulting and Clinical Psychology, 55, 635-644.

Polivy, J., \& Herman, C. P. (1991). Good and bad dieters: Self-perception and reaction to a dietary challenge. International Journal of Eating Disorders, 10, 91-99.

Polivy, J., \& Herman, C. P. (1993). Etiology of binge eating: Psychological mechanisms. In C. G. Fairburn \& G. T. Wilson (Eds.), Binge eating: Nature, assessment and treatment (pp. 173-205). New York: Guilford Press.

Polivy, J., \& Herman, C. P. (2002). Causes of eating disorders. Annual Review of Psychology, 53, 187-213.

Rosenthal, R., \& DiMatteo, M. R. (2001). Meta-analysis: Recent developments in quantitative methods for literature reviews. Annual Review of Psychology, 52, 59-82.

Ruderman, A. J., Belzer, L. J., \& Halperin, A. (1985). Restraint, anticipated consumption, and overeating. Journal of Abnormal Psychology, 94, 547-555.

Schlundt, D. G., \& Johnson, W. G. (1990). Eating disorders: Assessment and treatment. Boston: Allyn \& Bacon.

Spencer, J. A., \& Fremouw, W. J. (1979). Binge eating as a function of restraint and weight classification. Journal of Abnormal Psychology, 88, 262-267.

Stice, E. (2001). A prospective test of the dual-pathway model of bulimic pathology: Mediating effects of dieting and negative affect. Journal of Abnormal Psychology, 110, 124-135. 
Stice, E., \& Agras, W. S. (1998). Predicting onset and cessation of bulimic behaviors during adolescence: A longitudinal grouping analysis. Behavior Therapy, 29, 257-276.

Tomarken, A. J., \& Kirschenbaum, D. S. (1984). Effects of plans for future meals on counter regulatory eating by restrained eaters. Journal of Abnormal Psychology, 93, 458-472.
Watson, D., Clark, L. A., \& Tellegen, A. (1988). Development and validation of brief measures of positive and negative affect: The PANAS scales. Journal of Personality and Social Psychology, 54, 1063-1070.

Wilson, G. T. (1993). Relation of dieting and voluntary weight loss to psychological functioning and binge eating. Annals of Internal Medicine, 119, 727-730.

\section{Appendix}

\section{[University of Toronto Letterhead]}

\section{UNIVERSITY OF TORONTO STUDENT MEAL PLAN \\ (Low-Fat, Calorie-Reduced Weight Loss Program)}

Breakfast-1 Slim-Fast ${ }^{\mathrm{TM}}$ breakfast shake

Dinner-1 pre-packaged frozen Lean Cuisine ${ }^{\mathrm{TM}}$

Lunch -1 serving lean protein, 1 serving starch, 1 fruit or vegetable, 6 oz. skim milk

Snacks-2 per day

6-8 eight-oz. glasses of water per day (one or two may be replaced by a diet soda)

Lunch Servings

Lean protein suggestions:

30 grams (two slices) cooked turkey or chicken breast (no skin)

Starch suggestions:

1 slice white sandwich bread

$1 \frac{1}{2}$ slices of whole wheat bread

10 wheat or vegetable crackers

3 rice cake patties

50 grams cooked white rice

1 raw potato

$1 / 2$ cooked potato

$250 \mathrm{ml}$ of macaroni

Added fats (butter, margarine)

Ice cream

Chocolate

Baked goods (cookies, cakes, pie)

Potato chips

Candy

Fried foods (fries, chicken fingers)

Soft drinks

Submarine sandwiches
$1 / 2$ can of tuna or salmon

1 vegetarian meat patty

25 grams (one slice) cooked ham

25 grams (one chop) of pork

20 grams (one patty) lean ground beef

40 grams tofu

( $1 / 2$ of any of the above portions along with 20 grams cheddar cheese)

2 egg whites (no yolks)

Low-calorie salad dressings for iceberg lettuce
Snack possibilities:

Rice cakes

Low-fat yogurt (no fruit)

Apple, banana, orange, pear, plum

2 carrot sticks

3 celery sticks

Whole wheat or vegetable crackers

One small wheat or bran muffin

Popcorn (no butter)/pretzels

\section{PROHIBITED FOODS}

Cheese slices

Oils (cooking, salad dressing)

Cream sauces

Hamburgers

Cream, whole fat or $2 \%$ milk

Bacon

Egg yolks

Pizza

Exotic cheeses
Received January 11, 2001

Revision received November 30, 2001

Accepted December 10, 2001 